Journal of Applied Pharmaceutical Science Vol. 5 (12), pp. 008-013, December, 2015

Available online at http://www.japsonline.com

DOI: $10.7324 / \mathrm{JAPS} .2015 .501202$

ISSN 2231-3354 (cc) BY-NC-SA

\title{
Determination of the Chemical Stability of Various Formulations of Tobramycin Eye-Drops by HPLC Method and Data Analysis by R- GUI Stability Software
}

\author{
María Ana Rosasco, Adriana Inés Segall* \\ Cátedra de Calidad de Medicamentos, Facultad de Farmacia y Bioquímica, Universidad de Buenos Aires, CONICET, Junín 956, 1113 Buenos Aires, \\ Argentina.
}

\begin{tabular}{|c|c|}
\hline ARTICLE INFO & ABSTRACT \\
\hline $\begin{array}{l}\text { Article history: } \\
\text { Received on: } 28 / 09 / 2015 \\
\text { Revised on: } 10 / 10 / 2015 \\
\text { Accepted on: } 22 / 10 / 2015 \\
\text { Available online: } 27 / 12 / 2015\end{array}$ & $\begin{array}{l}\text { Tobramycin, is a water soluble aminoglycoside antibiotic produced by the fungus Streptomyces tenebrarius and } \\
\text { used in a variety of pharmaceutical applications including ophthalmic solutions, suspensions and ointments; } \\
\text { inhalation solutions and intravenous administration. There are commercially available eye drops formulations in } \\
\text { the Argentinian market that have different conservation conditions. We formulated six eye drops solutions, } \\
\text { studied their stability at } 2-8^{\circ} \mathrm{C}, 25^{\circ} \mathrm{C} \text {, and } 40^{\circ} \mathrm{C}, 75 \% \mathrm{RH} \text { and quantified using USP method. Only half of the }\end{array}$ \\
\hline $\begin{array}{l}\text { Key words: } \\
\text { Tobramycin, eyes-drops, } \\
\text { stability, software generated } \\
\text { shelf life, formulations. }\end{array}$ & $\begin{array}{l}\text { date and only one for three years in the same condition. One system was unstable in the three conservation } \\
\text { conditions studied, including appearance and } \mathrm{pH} \text {. }\end{array}$ \\
\hline
\end{tabular}

\section{INTRODUCTION}

Tobramycin, D-streptamine, $O-3$-amino-3-deoxy- $\alpha$-Dglucopyranosyl- $(1 \rightarrow 6)$ - $O$-['2,6-diamino-2,3,6-trideoxy- $\alpha$-Dribo-hexopyranosyl- $(1 \rightarrow 4)]-2$-deoxy-, is a water soluble aminoglycoside antibiotic produced by the fungus Streptomyces tenebrarius and used in a variety of pharmaceutical applications including ophthalmic solutions, suspensions and ointments; inhalation solutions and intravenous administration (Hanko and Rohrer, 2006; Manyanga et al., 2013). It is active against a broad spectrum of gram-negative bacteria. There are commercially available eye drops formulations in the Argentinian market that have different conservation conditions (ambient temperature and refrigerator). Brandl and $\mathrm{Gu}$ studied the stability of Tobramycin in aqueous solution (Brandl and Gu, 1992). They concluded that although hydrolysis of tobramycin occurs at the $\mathrm{pH}$ extremes, it is not an important degradation pathway at neutral $\mathrm{pH}$ values. The major degradation pathway for tobramycin at neutral $\mathrm{pH}$ values where the drug is formulated $(\mathrm{pH} \mathrm{5.8-7.4)}$ is oxidation.

* Corresponding Author

Email: asegall@ffyb.uba.ar
Tobramycin oxidizes giving several products including nebramine, deoxystreptamine and deoxystreptamine-kanasaminide (Fig 1). According to these results we formulated six solutions and studied their stability at $2-8^{\circ} \mathrm{C}, 25^{\circ} \mathrm{C}$, and $40^{\circ} \mathrm{C}, 75 \% \mathrm{RH}$. Direct analysis of tobramycin is not simple. This is due to the polar basic nature and the lack of UV absorbing chromophore in the molecule. Several methods have been described to determine tobramycin by adsorptive stripping voltammetric method (Sun et al., 2005), electrophoresis capillary (El-Attug et al., 2012; Ahmed and Ebeid, 2015) and ion pair LC methods (Hankoand Rohrer, 2006; Szúnyog et al., 2000; Valentini et al., 2008; Hanko et al., 2008; Chopra et al., 2010). A literature survey revealed some reversed phase liquid chromatographic methods for the quantitation of tobramycin employing mass detector (Keevil et al., 2003; Guo et al., 2006), ELSD (Megoulas et al., 2005; Pfeifer et al., 2015) and PED (Manyanga et al., 2013). The literature showed also reversed phase HPLC methods with pre column derivatization with 1 Naphthyl isothiocyanate (Feng et al., 2002), Fluorescein isothiocyanate (Mashat et al., 2008), 2,4,6-Trinitrobenzenesulphonic acid (Dash and Suryanarayanan, 1991a; Dash and Suryanarayanan, 1991b), 4Chloro-3,5-dinitrobenzotrifluoride (He et al., 2011), 9-Fluorenylmethyl chloroformate (Zhang and Peng, 2012) and 
<smiles>NC[C@H]1O[C@@H](O[C@@H]2[C@@H](N)C[C@@H](N)[C@H](O[C@@H]3OC(CO)[C@@H](O)[C@H](N)[C@H]3O)[C@H]2O)[C@H](N)C[C@H]1O</smiles>

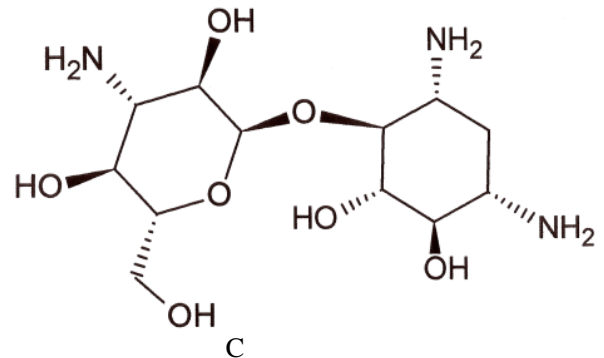<smiles>N[C@H]1C[C@@H](N)[C@H](O)[C@H](O)[C@H]1O</smiles><smiles>NC[C@H]1O[C@H](O[C@@H]2[C@@H](N)C[C@@H](N)[C@H](O)[C@H]2O)[C@H](N)C[C@H]1O</smiles>

Fig. 1: A) Tobramycin, B) Deoxystreptamine, C) Deoxystreptamine-kanasaminide, D) Nebramine.

Table 1: Quantitative composition of formulations ( $\mathrm{g} \%$ )

\begin{tabular}{|c|c|c|c|c|c|c|}
\hline Materials (g/100g) & System A & SystemB & System C & SystemD & SystemE & SystemF \\
\hline Tobramicyn & 0.3 & 0.3 & 0.3 & 0.3 & 0.3 & 0.3 \\
\hline Excipients:Benzalkonium chloride & $1.0 \times 10^{-2}$ & & & & & \\
\hline Sodium chloride & $1.0 \times 10^{-2}$ & 0.1 & $7.0 \times 10^{-2}$ & $2.0 \times 10^{-2}$ & & \\
\hline Dibasic sodium phosphate & 0.6 & & & & & \\
\hline Monobasic sodium phosphate & 0.4 & & & & & \\
\hline Boric acid & & $1.24 \times 10^{-2}$ & & $1.26 \times 10^{-3}$ & 1.9 & 1.9 \\
\hline Anhydrous sodium sulfate & & $1.52 \times 10^{-3}$ & & $2.26 \times 10^{-4}$ & & \\
\hline Tyloxapol & & $1.0 \times 10^{-3}$ & & 0.1 & & \\
\hline Edetate disodium & & & 0.1 & & & \\
\hline Sodium metabisulfite & & & 0.5 & & & \\
\hline Thimerosal & & & $1.0 \times 10^{-3}$ & & & \\
\hline Sodium hyaluronate & & & & & 0.3 & 0.3 \\
\hline Potassium sorbate & & & & & & 0.18 \\
\hline Water for injection csp & 100 & 100 & 100 & 100 & 100 & 100 \\
\hline
\end{tabular}

o-Phthalaldehyde pre column derivatization (Caturla et al., 1992) and post column derivatization (He and Yang, 2010). This work describes the analysis of tobramycin in six formulations maintained in stability studies and quantitated using USP method (The United States Pharmacopeia, 2015). This method use 2,4Dinitrofluorobenzene as pre- derivatizating agent. Russ and coworkers use a modification of this method for the quantitation of tobramicyn in an ophthalmic suspension (Russ et al., 1998).

\section{MATERIALS AND METHODS}

\section{Materials and reagents}

Tobramicyn $(955,9 \mu \mathrm{g} / \mathrm{g}$ calculated with reference to the dried substance) pharmaceutical grade was provided by Cross Chem (China), Benzalkonium chloride Merck (Germany), Sodium chloride Merck (USA), Dibasic sodium phosphate Anedra (Argentine), Monobasic sodium phosphate Anedra (Argentine), Boric acid J.T Baker (Mexico), Anhydrous sodium sulfate Anedra
(Argentine), Tyloxapol Sigma-Aldrich (India), Edetate disodium Merck (Germany), Sodium hydroxide Anedra (Argentine), Sodium metabisulfite J.T. Baker (Mexico), Thimerosal Ningbo Hi-Tech (China), Sodium hyaluronate CPN SPOL SRO (Czech Republic), Potassium sorbate granular Merck (Germany) and distilled water.

All chemicals used were of analytical grade, sulphuric acid Merck (Germany), Buffer TRIS (Tris(Hidroxymethyl) aminomethane) Biopack (Argentine), 2,4-Dinitrofluorobenzene Sigma-Aldrich (USA), p-naphtholbenzein Sigma-Aldrich (USA). Acetonitrile and water were of HPLC grade. Solvents were filtered through a $0.45 \mu \mathrm{m}$ membrane and degassed.

\section{Preparation of the eye-drops}

The six systems were prepared with the same drug substance of Tobramycin. Formulations are described in Table 1. In a glass flask containing $350 \mathrm{~mL}$ of distilled water at $25^{\circ} \mathrm{C}$, the salts and other excipients were dissolved once each and stirring 
vigorously. A nitrogen flow was placed before the Tobramycin was added. Then, the $\mathrm{pH}$ was corrected around 7.5 with $\mathrm{NaOH} 1 \mathrm{~N}$ or $\mathrm{HCl} 3 \mathrm{~N}$. The solutions were diluted with distilled water to 500 $\mathrm{mL}$. The solutions were passed through a $0.22 \mu \mathrm{m}$ nylon membrane filter before injection $\left(25 \mathrm{~mm}\right.$ disposable filter; Cat. $\mathrm{N}^{\circ}$ R04SP02500 Osmonics Inc., Minnesota, USA). Approximately 5 $\mathrm{mL}$ of Tobramycin solutions were transfer in plastic bottles of HDPE/LDPE and placed in stability conditions: $2-8{ }^{\circ} \mathrm{C}, 25^{\circ} \mathrm{C}$ and $40{ }^{\circ} \mathrm{C}, 75 \% \mathrm{HR}$.

\section{Instrumentation}

The HPLC system consisted of a dual piston reciprocating Thermo Finnigan pump (Waltham, Massachusetts, United States, Model P2000), a Rheodyne injector (Model 7125), a UV-Vis KONIK detector (Barcelona, Spain, Model KNK-027757) with operating software WinPCC Chrom XY (Buenos Aires, Argentine) was used during the study.

\section{HPLC conditions}

The experiment was performed on a reversed phase $\mathrm{C} 18$ column (Phenomenex, Torrance, CA, USA) $300 \mathrm{x} 4.6 \mathrm{~mm}, 10 \mu \mathrm{m}$. The separation was carried out under isocratic elution with acetonitrile:buffer TRIS. For the preparation of the mobile phase, $2 \mathrm{~g}$ of buffer TRIS were dissolved in $800 \mathrm{ml}$ of water. To this solution, $20 \mathrm{ml}$ of Sulfuric acid was added and diluted to $2000 \mathrm{ml}$ with acetonitrile. The flow rate was $1.1 \mathrm{~mL} / \mathrm{min}$. The wavelength was monitored at $365 \mathrm{~nm}$, and the injection volume was $20 \mu \mathrm{L}$. The HPLC was operated at ambient temperature. In these conditions the retention time $\left(t_{R}\right)$ was roughly 6 minutes.

\section{Preparation of standard solution}

An accurately weighed quantity of $33 \mathrm{mg}$ of tobramycin was transferred to a $50 \mathrm{~mL}$ volumetric flask, added $1 \mathrm{~mL}$ of $1 \mathrm{~N}$ sulfuric acid and dissolved in $20 \mathrm{ml}$ of water and then taken to volume with water. Then, $10.0 \mathrm{~mL}$ were withdraw in a $50 \mathrm{~mL}$ volumetric flask, diluted with water to volume, and mixed. The solutions were passed through a $0.45 \mu \mathrm{m}$ nylon membrane filter before injection (25 mm disposable filter; Cat. $\mathrm{N}^{\circ}$ Y02025WPH $\mu$ icroclar, Buenos Aires, Argentina).

\section{Sample Preparation}

Approximately $1 \mathrm{~mL}$ of eye drops tobramycin solution $0.3 \%$ ( $3 \mathrm{mg} / \mathrm{mL}$ ) were exactly weighed, placed into a $25 \mathrm{~mL}$ volumetric flask and taken to volume with water.

The solutions were passed through a $0.45 \mu \mathrm{m}$ nylon membrane filter before injection (25 mm disposable filter; Cat. $\mathrm{N}^{\circ}$ Y02025WPH $\mu$ icroclar, Buenos Aires, Argentina).

\section{Derivatization}

A solution of 2,4-Dinitrofluorobenzene containing $10 \mathrm{mg}$ per $\mathrm{mL}$ in alcohol was prepared and maintained during 5 days in refrigerator. A stock solution of TRIS was prepared in water containing $15 \mathrm{mg}$ per mL. $40 \mathrm{~mL}$ were transferred to a $200 \mathrm{~mL}$ volumetric flask and diluted to volume with dimethyl sulfoxide.
This reagent was used within $4 \mathrm{hs}$. To separate $50 \mathrm{~mL}$ volumetric flask, $4.0 \mathrm{~mL}$ of standard preparation, $4.0 \mathrm{~mL}$ of sample preparation and $4.0 \mathrm{~mL}$ of water were transferred and added 10 $\mathrm{mL}$ of 2,4-Dinitrofluorobenzene solution and $10 \mathrm{~mL}$ of TRIS solution each. The volumetric flask were kept in a water bath at 60 $\pm 2{ }^{\circ} \mathrm{C}$ during $50 \pm 5$ minutes. The flask were removed from the bath, and allowed to stand for 10 minutes. Added acetonitrile to about $2 \mathrm{~mL}$ below the $50 \mathrm{~mL}$ mark, allowed to cool to room temperature, then diluted with acetonitrile to volume, and mixed.

The resolution solution was prepared transferring $2 \mathrm{~mL}$ of a fresh solution of p-naphtholbenzein in acetonitrile (containing $0.24 \mathrm{mg}$ per $\mathrm{mL}$ ) to a $10 \mathrm{~mL}$ volumetric flask and diluted with derivatized standard preparation for volume and used promptly.

It was injected a blank of mobile phase, another with the derivatization solution diluted as the standard solution and the resolution solution. The relative retention times were about 0.6 for p-naphtholbenzein and 1.0 for tobramycin, and the resolution, R, between the two peaks was not less 4.0.The derivatized standard preparation was chromatographed, recorded the responses until the relative standard deviation for replicate was not more than $2.0 \%$. Separately were injected equal volumes of the derivatized standard preparation and the derivatized assay preparation into the chromatograph, recorded the chromatograms and measured the area responses for the major peaks and calculated the quantity.

\section{pH Determinations}

The $\mathrm{pH}$ data for all the systems were obtained with model Altronix TPX I (Saen S.R.L., Buenos Aires, Argentina). The $\mathrm{pH}$ was measured as directed in USP $38<791>$, using an indicator glass electrode. The buffer solutions for standardization were from Merck (Darmstadt, Germany) at pH 4.00 and 7.01.

\section{Software}

To evaluate the shelf life of Tobramycin eye drops, an Rpackage "stab" software version 3.0.1 was used (Lee et al., 2010; Hassan et al., 2015). The software meets ICH Q1E specifications (Guidance for the industry: ICH 2003).

\section{RESULTS AND DISCUSSION}

In the present study, the stability of different eye drops formulations containing Tobramycin were studied. The eye drops produced in this work are similar to those it can be found in the Argentinian market. The trouble is that the products have different conservation conditions.

The stability studies are executed to estimate the mechanism of the active pharmaceutical ingredients' (APIs) degradation in crude and in dosage forms.

The preparations were analyzed by HPLC with a previous derivatization reaction and UV detection. The stability results were analysed with R-package "stab" software version 3.0.1., having a single-factor analysis, for single-batch based on ICH Q1E specifications (Guidance for the industry: ICH 2003). The assay results are mentioned in Table 2 . With reference to $\mathrm{pH}$ for tobramycin eye-drops solutions, USP 38 (The United States 
Pharmacopeia, 2015) sets a range from 7 to 8 . The appearance and $\mathrm{pH}$ results are indicated in Table 3 , in bold $\mathrm{pH}$ data that are out of specification. Only system $\mathrm{C}$ does not meet this requirement in any of the storage conditions. First order analysis was made at one sided lower control analysis at $90 \%$ confidence interval (Fig. 2-4). The results are presented in Table 4 . In refrigerator $\left(2-8{ }^{\circ} \mathrm{C}\right)$ systems A, B, D and E shown a shelf life of 5 years or more while system $\mathrm{F}$ present 4.83 years and system $\mathrm{C} 1.58$ years. At ambient

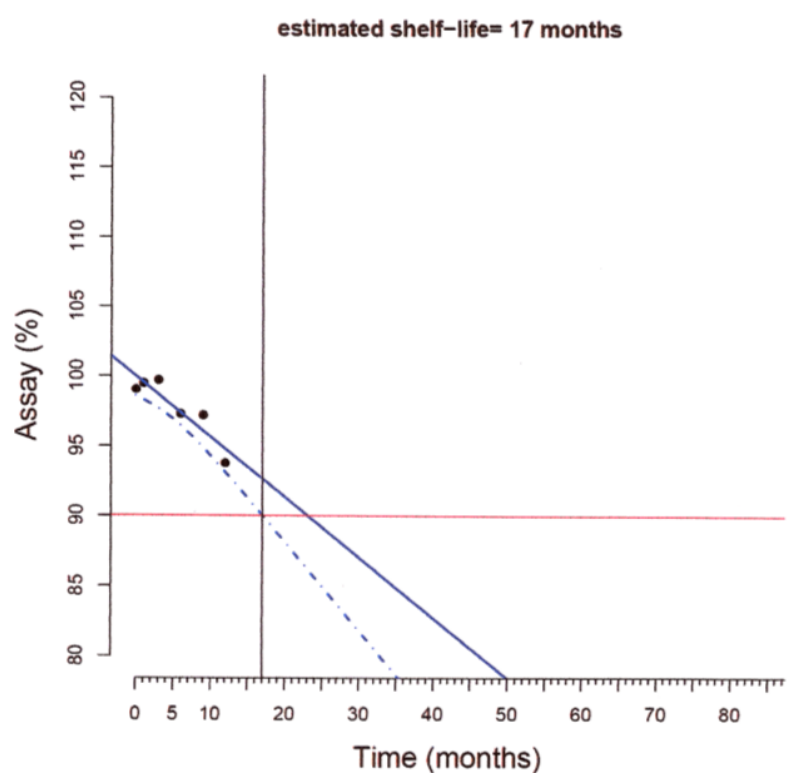

Fig. 2: Estimation of shelf life of Tobramycin System $\mathrm{D}$ al $40{ }^{\circ} \mathrm{C}, 75 \% \mathrm{RH}$ temperature $\left(25^{\circ} \mathrm{C}\right)$, systems B was the most stable with 4.58 years and systems $\mathrm{D}$ and $\mathrm{E}$ greater than 24 months. At the accelerated condition $\left(40{ }^{\circ} \mathrm{C}\right.$ and $\left.75 \% \mathrm{RH}\right)$, only system B was stable at 1.75 years. System B was found most stable whereas system $\mathrm{C}$ was the least stable. With regard to appearance, System $\mathrm{C}$ seems to turn to yellowish at 6 months at ambient temperature. The same occur to System F since 9 months and System A since 18 months.

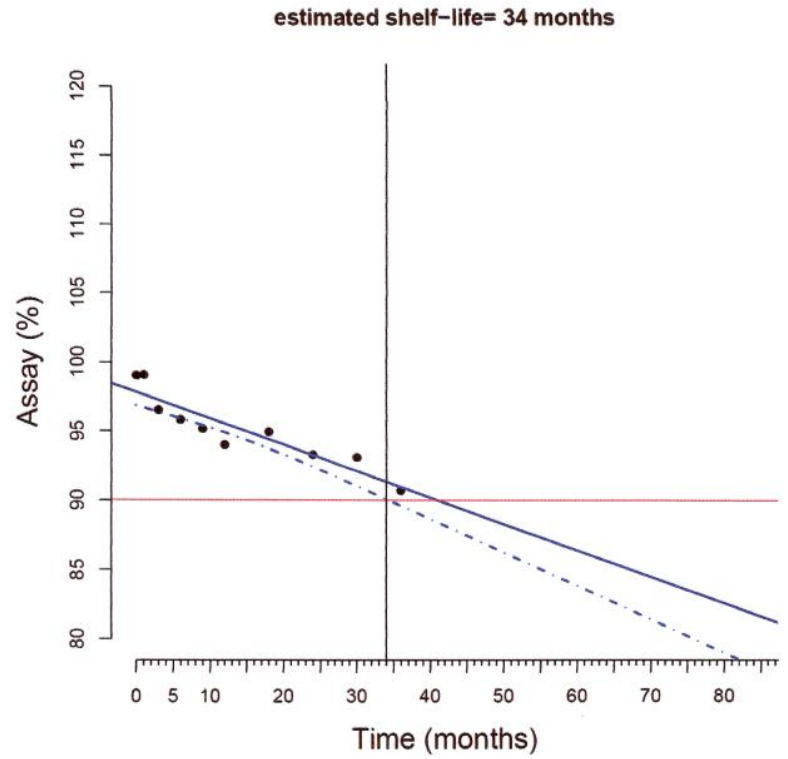

Fig. 3: Estimation of shelf life of Tobramycin System $\mathrm{D}$ al $25^{\circ} \mathrm{C}$.

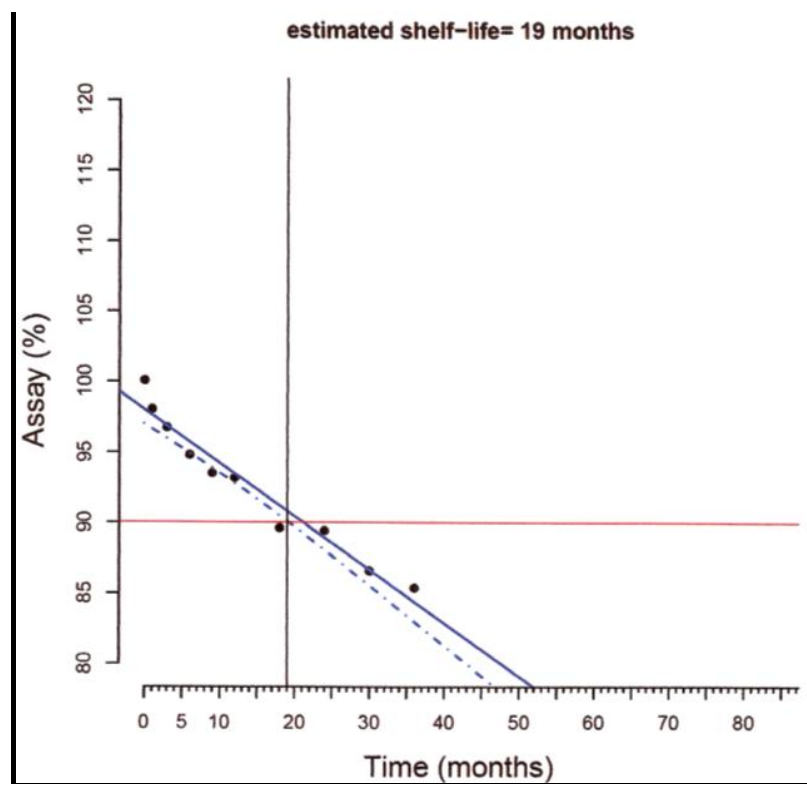

Fig. 4: Estimation of shelf life of Tobramycin System $\mathrm{C}$ al $2-8^{\circ} \mathrm{C}$. 
Table 2: The remaining percentage content of the active ingredient tobramycin in eye-drops solutions.

\begin{tabular}{|c|c|c|c|c|c|c|c|c|c|c|}
\hline \multicolumn{11}{|c|}{ Time (months) } \\
\hline System A & $\mathbf{0}$ & 1 & 3 & 6 & 9 & 12 & 18 & 24 & 30 & 36 \\
\hline $40^{\circ} \mathrm{C}, 75 \% \mathrm{HR}$ & 97.65 & 93.08 & 88.19 & 88.32 & 74.52 & 62.92 & & & & \\
\hline $25^{\circ} \mathrm{C}, 60 \% \mathrm{HR}$ & 97.65 & 99.92 & 96.24 & 95.11 & 95.84 & 94.77 & 91.50 & 91.45 & 85.95 & 85.30 \\
\hline $2-8^{\circ} \mathrm{C}$ & 97.65 & 98.48 & 96.84 & 96.71 & 97.50 & 97.24 & 97.25 & 98.47 & 98.24 & 99.54 \\
\hline \multicolumn{11}{|l|}{ SystemB } \\
\hline $40^{\circ} \mathrm{C}, 75 \% \mathrm{HR}$ & 99.42 & 97.09 & 98.87 & 95.90 & 96.70 & 96.47 & & & & \\
\hline $25^{\circ} \mathrm{C}, 60 \% \mathrm{HR}$ & 99.42 & 100.94 & 97.56 & 95.67 & 97.63 & 98.08 & 97.38 & 99.12 & 95.29 & 94.51 \\
\hline $2-8^{\circ} \mathrm{C}$ & 99.42 & 97.41 & 96.72 & 96.66 & 97.73 & 98.15 & 98.09 & 98.48 & 99.86 & 98.99 \\
\hline \multicolumn{11}{|l|}{ SystemC } \\
\hline $40^{\circ} \mathrm{C}, 75 \% \mathrm{HR}$ & 100.09 & 82.42 & 80.11 & 75.40 & 73.82 & 68.78 & & & & \\
\hline $25^{\circ} \mathrm{C}, 60 \% \mathrm{HR}$ & 100.09 & 94.32 & 84.70 & 80.53 & 80.82 & 79.56 & 77.70 & 75.87 & 76.06 & 69.97 \\
\hline $2-8^{\circ} \mathrm{C}$ & 100.09 & 98.08 & 96.78 & 94.84 & 93.55 & 93.18 & 89.65 & 89.48 & 86.62 & 85.43 \\
\hline \multicolumn{11}{|l|}{ SystemD } \\
\hline $40^{\circ} \mathrm{C}, 75 \% \mathrm{HR}$ & 99.04 & 99.48 & 99.71 & 97.30 & 97.19 & 93.78 & & & & \\
\hline $25^{\circ} \mathrm{C}, 60 \% \mathrm{HR}$ & 99.04 & 99.09 & 96.52 & 95.84 & 95.16 & 94.00 & 94.95 & 93.27 & 93.12 & 90.72 \\
\hline $2-8^{\circ} \mathrm{C}$ & 99.04 & 97.41 & 97.12 & 95.77 & 96.73 & 97.17 & 97.53 & 97.46 & 97.53 & 96.56 \\
\hline \multicolumn{11}{|l|}{ SystemE } \\
\hline $40^{\circ} \mathrm{C}, 75 \% \mathrm{HR}$ & 99.24 & 97.20 & 97.54 & 88.70 & 85.77 & 81.49 & & & & \\
\hline $25^{\circ} \mathrm{C}, 60 \% \mathrm{HR}$ & 99.24 & 100.77 & 96.29 & 96.31 & 96.24 & 93.47 & 95.56 & 95.88 & 90.03 & 87.55 \\
\hline $2-8^{\circ} \mathrm{C}$ & 99.24 & 97.58 & 96.75 & 96.34 & 96.53 & 96.52 & 97.27 & 99.04 & 97.87 & 100.74 \\
\hline \multicolumn{11}{|l|}{ SystemF } \\
\hline $40^{\circ} \mathrm{C}, 75 \% \mathrm{HR}$ & 98.6 & 92.18 & 93.61 & 86.68 & 81.30 & 69.08 & & & & \\
\hline $25^{\circ} \mathrm{C}, 60 \% \mathrm{HR}$ & 98.6 & 98.70 & 94.62 & 92.79 & 93.24 & 91.88 & 90.48 & 84.73 & 85.73 & 83.82 \\
\hline $2-8^{\circ} \mathrm{C}$ & 98.6 & 96.04 & 96.07 & 95.30 & 95.80 & 94.99 & 95.45 & 94.80 & 93.12 & 95.52 \\
\hline
\end{tabular}

Table 3: Appearance and $\mathrm{pH}$ of the formulations.

\begin{tabular}{|c|c|c|c|c|c|c|c|c|c|c|c|c|c|c|c|c|c|c|c|}
\hline & & Syst & n A & & Syst & A B & & Syst & $\mathrm{C}$ & & Syst & $\mathrm{nD}$ & & Syst & $\mathbf{n E}$ & & Syste & & \\
\hline $\begin{array}{c}\text { Time } \\
\text { (month) }\end{array}$ & & $\begin{array}{l}\mathbf{2 - 8} \\
{ }^{\circ} \mathrm{C}\end{array}$ & $25^{\circ} \mathrm{C}$ & $40^{\circ} \mathrm{C}$ & $\begin{array}{l}2-8 \\
{ }^{\circ} \mathrm{C}\end{array}$ & $25^{\circ} \mathrm{C}$ & $40^{\circ} \mathrm{C}$ & $\begin{array}{l}2-8 \\
{ }^{\circ} \mathrm{C}\end{array}$ & $25^{\circ} \mathrm{C}$ & $40^{\circ} \mathrm{C}$ & $\begin{array}{c}2- \\
8^{\circ} \mathrm{C}\end{array}$ & $25^{\circ} \mathrm{C}$ & $40^{\circ} \mathrm{C}$ & $\begin{array}{c}2- \\
8^{\circ} \mathrm{C}\end{array}$ & $\begin{array}{l}25 \\
{ }^{\circ} \mathrm{C}\end{array}$ & $40^{\circ} \mathrm{C}$ & $\begin{array}{c}2- \\
8^{\circ} \mathrm{C}\end{array}$ & $25^{\circ} \mathrm{C}$ & $40^{\circ} \mathrm{C}$ \\
\hline 0 & $\begin{array}{l}\text { Appearance } \\
\mathrm{pH}\end{array}$ & $\begin{array}{c}\mathrm{a} \\
7.52\end{array}$ & $\begin{array}{c}\mathrm{a} \\
7.52\end{array}$ & $\begin{array}{c}\mathrm{a} \\
7.52\end{array}$ & $\begin{array}{c}a \\
7.50\end{array}$ & $\begin{array}{c}\mathrm{a} \\
7.50\end{array}$ & $\begin{array}{c}\mathrm{a} \\
7.50\end{array}$ & $\begin{array}{c}a \\
7.55\end{array}$ & $\begin{array}{c}\mathrm{a} \\
7.55\end{array}$ & $\begin{array}{c}\mathrm{a} \\
7.55\end{array}$ & $\begin{array}{c}\mathrm{a} \\
7.40\end{array}$ & $\begin{array}{c}\mathrm{a} \\
7.40\end{array}$ & $\begin{array}{c}\mathrm{a} \\
7.40\end{array}$ & $\begin{array}{c}\mathrm{a} \\
7.42\end{array}$ & $\begin{array}{c}a \\
7.42\end{array}$ & $\begin{array}{c}\mathrm{a} \\
7.42\end{array}$ & $\begin{array}{c}\mathrm{a} \\
7.40\end{array}$ & $\begin{array}{c}\mathrm{a} \\
7.40\end{array}$ & $\begin{array}{c}\mathrm{a} \\
7.40\end{array}$ \\
\hline 1 & $\begin{array}{l}\text { Appearance } \\
\mathrm{pH}\end{array}$ & $\begin{array}{c}\mathrm{a} \\
7.69\end{array}$ & $\begin{array}{c}\mathrm{a} \\
7.69\end{array}$ & $\begin{array}{c}\mathrm{a} \\
7.68 \\
\end{array}$ & $\begin{array}{c}\mathrm{a} \\
7.50\end{array}$ & $\begin{array}{c}\mathrm{a} \\
7.50\end{array}$ & $\begin{array}{c}\mathrm{a} \\
7.49\end{array}$ & $\begin{array}{c}\mathrm{a} \\
7.48 \\
\end{array}$ & $\begin{array}{c}\mathrm{a} \\
7.23 \\
\end{array}$ & $\begin{array}{c}\mathrm{c} \\
6.98 \\
\end{array}$ & $\begin{array}{c}\mathrm{a} \\
7.37\end{array}$ & $\begin{array}{c}\mathrm{a} \\
7.33\end{array}$ & $\begin{array}{c}\mathrm{a} \\
7.37\end{array}$ & $\begin{array}{c}\mathrm{a} \\
7.47\end{array}$ & $\begin{array}{c}\mathrm{a} \\
7.44\end{array}$ & $\begin{array}{c}\mathrm{a} \\
7.50\end{array}$ & $\begin{array}{c}\mathrm{a} \\
7.40\end{array}$ & $\begin{array}{c}\mathrm{a} \\
7.41\end{array}$ & $\begin{array}{c}\mathrm{c} \\
7.41\end{array}$ \\
\hline 3 & $\begin{array}{l}\text { Appearance } \\
\mathrm{pH}\end{array}$ & $\begin{array}{c}\mathrm{a} \\
7.69 \\
\end{array}$ & $\begin{array}{c}\mathrm{a} \\
7.68 \\
\end{array}$ & $\begin{array}{c}\mathrm{a} \\
7.68 \\
\end{array}$ & $\begin{array}{c}\mathrm{a} \\
7.51 \\
\end{array}$ & $\begin{array}{c}\mathrm{a} \\
7.53 \\
\end{array}$ & $\begin{array}{c}\mathrm{a} \\
7.52 \\
\end{array}$ & $\begin{array}{c}\mathrm{a} \\
7.40 \\
\end{array}$ & $\begin{array}{c}\mathrm{a} \\
7.17 \\
\end{array}$ & $\begin{array}{c}\mathrm{a} \\
6.97 \\
\end{array}$ & $\begin{array}{c}\mathrm{a} \\
7.33 \\
\end{array}$ & $\begin{array}{c}\mathrm{a} \\
7.34 \\
\end{array}$ & $\begin{array}{c}\mathrm{a} \\
7.34 \\
\end{array}$ & $\begin{array}{c}\mathrm{a} \\
7.49 \\
\end{array}$ & $\begin{array}{c}\mathrm{a} \\
7.49 \\
\end{array}$ & $\begin{array}{c}\mathrm{a} \\
7.50 \\
\end{array}$ & $\begin{array}{c}\mathrm{a} \\
7.43 \\
\end{array}$ & $\begin{array}{c}\mathrm{a} \\
7.42 \\
\end{array}$ & $\begin{array}{c}\mathrm{a} \\
7.40 \\
\end{array}$ \\
\hline 6 & $\begin{array}{l}\text { Appearance } \\
\mathrm{pH}\end{array}$ & $\begin{array}{c}\mathrm{a} \\
7.70 \\
\end{array}$ & $\begin{array}{c}\mathrm{a} \\
7.70 \\
\end{array}$ & $\begin{array}{c}\mathrm{a} \\
7.69 \\
\end{array}$ & $\begin{array}{c}\mathrm{a} \\
7.52 \\
\end{array}$ & $\begin{array}{c}\mathrm{a} \\
7.56 \\
\end{array}$ & $\begin{array}{c}\mathrm{a} \\
7.55 \\
\end{array}$ & $\begin{array}{c}\mathrm{a} \\
7.37 \\
\end{array}$ & $\begin{array}{c}\mathrm{c} \\
6.99 \\
\end{array}$ & $\begin{array}{c}\mathrm{d} \\
6.92 \\
\end{array}$ & $\begin{array}{c}\mathrm{a} \\
7.26 \\
\end{array}$ & $\begin{array}{c}\mathrm{a} \\
7.37 \\
\end{array}$ & $\begin{array}{c}\mathrm{a} \\
7.36 \\
\end{array}$ & $\begin{array}{c}\mathrm{a} \\
7.50 \\
\end{array}$ & $\begin{array}{c}\mathrm{a} \\
7.55 \\
\end{array}$ & $\begin{array}{c}\mathrm{a} \\
7.50 \\
\end{array}$ & $\begin{array}{c}\mathrm{a} \\
7.42 \\
\end{array}$ & $\begin{array}{c}\mathrm{a} \\
7.43 \\
\end{array}$ & $\begin{array}{c}\mathrm{c} \\
7.42 \\
\end{array}$ \\
\hline 9 & $\begin{array}{l}\text { Appearance } \\
\mathrm{pH}\end{array}$ & $\begin{array}{c}a \\
7.69\end{array}$ & $\begin{array}{c}\mathrm{a} \\
7.67\end{array}$ & $\begin{array}{c}c \\
7.59\end{array}$ & $\begin{array}{c}\mathrm{a} \\
7.44\end{array}$ & $\begin{array}{c}\mathrm{a} \\
7.42\end{array}$ & $\begin{array}{c}\mathrm{a} \\
7.45\end{array}$ & $\begin{array}{c}\mathrm{a} \\
7.33\end{array}$ & $\begin{array}{c}\mathrm{d} \\
6.90\end{array}$ & $\begin{array}{c}\mathrm{d} \\
6.78\end{array}$ & $\begin{array}{c}\mathrm{a} \\
7.16\end{array}$ & $\begin{array}{c}\mathrm{a} \\
7.19\end{array}$ & $\begin{array}{c}\mathrm{a} \\
7.23\end{array}$ & $\begin{array}{c}\mathrm{a} \\
7.52\end{array}$ & $\begin{array}{c}\mathrm{a} \\
7.45\end{array}$ & $\begin{array}{c}\mathrm{a} \\
7.47\end{array}$ & $\begin{array}{c}\mathrm{a} \\
7.44\end{array}$ & $\begin{array}{c}c c \\
7.39\end{array}$ & $\begin{array}{c}c \\
7.42\end{array}$ \\
\hline 12 & $\begin{array}{l}\text { Appearance } \\
\mathrm{pH}\end{array}$ & $\begin{array}{c}\mathrm{a} \\
7.63 \\
\end{array}$ & $\begin{array}{c}\mathrm{a} \\
7.65 \\
\end{array}$ & $\begin{array}{c}\mathrm{c} \\
7.49 \\
\end{array}$ & $\begin{array}{c}\mathrm{a} \\
7.30 \\
\end{array}$ & $\begin{array}{c}\mathrm{a} \\
7.39 \\
\end{array}$ & $\begin{array}{c}\mathrm{a} \\
7.40 \\
\end{array}$ & $\begin{array}{c}\mathrm{a} \\
7.19 \\
\end{array}$ & $\begin{array}{c}\mathrm{d} \\
6.85 \\
\end{array}$ & $\begin{array}{c}\mathrm{d} \\
6.62 \\
\end{array}$ & $\begin{array}{c}\mathrm{a} \\
7.11 \\
\end{array}$ & $\begin{array}{c}\mathrm{a} \\
7.17\end{array}$ & $\begin{array}{c}\mathrm{c} \\
7.15\end{array}$ & $\begin{array}{c}\mathrm{a} \\
7.47 \\
\end{array}$ & $\begin{array}{c}\mathrm{a} \\
7.47 \\
\end{array}$ & $\begin{array}{c}\mathrm{c} \\
7.44\end{array}$ & $\begin{array}{c}\mathrm{a} \\
7.40 \\
\end{array}$ & $\begin{array}{c}\mathrm{c} \\
7.41 \\
\end{array}$ & $\begin{array}{c}\mathrm{c} \\
7.34 \\
\end{array}$ \\
\hline 18 & $\begin{array}{l}\text { Appearance } \\
\mathrm{pH}\end{array}$ & $\begin{array}{c}\mathrm{a} \\
7.67 \\
\end{array}$ & $\begin{array}{c}\mathrm{b} \\
7.65 \\
\end{array}$ & $\begin{array}{l}- \\
- \\
\end{array}$ & $\begin{array}{c}\mathrm{a} \\
7.51 \\
\end{array}$ & $\begin{array}{c}\mathrm{a} \\
7.41 \\
\end{array}$ & $\begin{array}{l}- \\
- \\
\end{array}$ & $\begin{array}{c}\mathrm{a} \\
7.15 \\
\end{array}$ & $\begin{array}{c}\mathrm{d} \\
6.85 \\
\end{array}$ & $\begin{array}{l}- \\
- \\
\end{array}$ & $\begin{array}{c}\mathrm{a} \\
7.24 \\
\end{array}$ & $\begin{array}{c}\mathrm{a} \\
7.17\end{array}$ & $\begin{array}{l}- \\
- \\
\end{array}$ & $\begin{array}{c}\mathrm{a} \\
7.57 \\
\end{array}$ & $\begin{array}{c}\mathrm{a} \\
7.44 \\
\end{array}$ & $\begin{array}{l}- \\
- \\
\end{array}$ & $\begin{array}{c}\mathrm{a} \\
7.48 \\
\end{array}$ & $\begin{array}{c}\mathrm{c} \\
7.34 \\
\end{array}$ & $\begin{array}{l}- \\
- \\
\end{array}$ \\
\hline 24 & $\begin{array}{l}\text { Appearance } \\
\mathrm{pH}\end{array}$ & $\begin{array}{c}\mathrm{a} \\
7.67 \\
\end{array}$ & $\begin{array}{c}\mathrm{c} \\
7.65 \\
\end{array}$ & - & $\begin{array}{c}\mathrm{b} \\
7.45 \\
\end{array}$ & $\begin{array}{c}\mathrm{a} \\
7.52 \\
\end{array}$ & - & $\begin{array}{c}\mathrm{a} \\
7.03 \\
\end{array}$ & $\begin{array}{c}\mathrm{d} \\
6.96 \\
\end{array}$ & - & $\begin{array}{c}\mathrm{a} \\
7.14 \\
\end{array}$ & $\begin{array}{c}\mathrm{a} \\
7.27 \\
\end{array}$ & - & $\begin{array}{c}\mathrm{a} \\
7.53 \\
\end{array}$ & $\begin{array}{c}\mathrm{a} \\
7.50 \\
\end{array}$ & - & $\begin{array}{c}\mathrm{a} \\
7.42 \\
\end{array}$ & $\begin{array}{c}\mathrm{c} \\
7.41 \\
\end{array}$ & - \\
\hline 30 & $\begin{array}{l}\text { Appearance } \\
\mathrm{pH}\end{array}$ & $\begin{array}{c}\mathrm{a} \\
7.66\end{array}$ & $\begin{array}{c}c \\
7.63 \\
\end{array}$ & $\begin{array}{l}- \\
-\end{array}$ & $\begin{array}{c}c \\
7.36 \\
\end{array}$ & $\begin{array}{c}\mathrm{a} \\
7.47 \\
\end{array}$ & $\begin{array}{l}- \\
-\end{array}$ & $\begin{array}{c}\mathrm{a} \\
6.84 \\
\end{array}$ & $\begin{array}{c}\mathrm{d} \\
6.86 \\
\end{array}$ & $\begin{array}{l}- \\
-\end{array}$ & $\begin{array}{c}\mathrm{a} \\
7.05\end{array}$ & $\begin{array}{c}\mathrm{a} \\
7.24 \\
\end{array}$ & - & $\begin{array}{c}\mathrm{a} \\
7.44 \\
\end{array}$ & $\begin{array}{c}\mathrm{a} \\
7.48 \\
\end{array}$ & $\begin{array}{l}- \\
-\end{array}$ & $\begin{array}{c}\mathrm{a} \\
7.38 \\
\end{array}$ & $\begin{array}{c}\mathrm{c} \\
7.41 \\
\end{array}$ & - \\
\hline 36 & $\begin{array}{l}\text { Appearance } \\
\mathrm{pH}\end{array}$ & $\begin{array}{c}\mathrm{a} \\
7.65\end{array}$ & $\begin{array}{c}c \\
7.61\end{array}$ & - & $\begin{array}{c}\mathrm{c} \\
7.32 \\
\end{array}$ & $\begin{array}{c}\mathrm{a} \\
7.43 \\
\end{array}$ & - & $\begin{array}{c}\mathrm{b} \\
6.77 \\
\end{array}$ & $\begin{array}{c}\mathrm{d} \\
6.75 \\
\end{array}$ & - & $\begin{array}{c}\mathrm{a} \\
7.08 \\
\end{array}$ & $\begin{array}{c}\mathrm{a} \\
7.19\end{array}$ & - & $\begin{array}{c}\mathrm{a} \\
7.51 \\
\end{array}$ & $\begin{array}{c}\mathrm{a} \\
7.48 \\
\end{array}$ & - & $\begin{array}{c}\mathrm{a} \\
7.48 \\
\end{array}$ & $\begin{array}{c}\mathrm{c} \\
7.42 \\
\end{array}$ & - \\
\hline
\end{tabular}

a: limpid solution, colorless., b: limpid solution, slightly yellowish., c: limpid solution, yellowish., d: limpid solution, yellow

Table 4: Software originated results for apparent First-Order Rate Constant $\left(\mathrm{k}_{\mathrm{obs}}\right)$ for the degradation of tobramycin eye-drops.

\begin{tabular}{lccc}
\hline System & $\mathrm{K}_{\text {obs. }}$ month $^{-1}$ & Correlationcoefficient & $\mathrm{t}_{90}$ years (months) \\
\hline $\mathrm{A} 40^{\circ} \mathrm{C}, 75 \% \mathrm{RH}$ & 2.655 & 0.9648 & $0.08(1 \mathrm{month})$ \\
$25{ }^{\circ} \mathrm{C}$ & 0.368 & 0.9679 & $1.67(20$ months $)$ \\
$2-8^{\circ} \mathrm{C}$ & 0.045 & 0.6497 & $>5$ \\
\hline $\mathrm{B} 40^{\circ} \mathrm{C}, 75 \% \mathrm{RH}$ & 0.204 & 0.6814 & $1.75(21 \mathrm{months})$ \\
$25{ }^{\circ} \mathrm{C}$ & 0.101 & 0.6401 & $4.58(55$ months $)$ \\
$2-8^{\circ} \mathrm{C}$ & 0.049 & 0.5786 & $>5$ \\
\hline $\mathrm{C} 40^{\circ} \mathrm{C}, 75 \% \mathrm{RH}$ & 1.970 & 0.8503 & $0.08(<1$ month $)$ \\
$25{ }^{\circ} \mathrm{C}$ & 0.598 & 0.8380 & $0.08(<1$ month) \\
$2-8^{\circ} \mathrm{C}$ & 0.378 & 0.9757 & $1.58(19$ months $)$ \\
\hline $\mathrm{D} 40^{\circ} \mathrm{C}, 75 \% \mathrm{RH}$ & 0.431 & $1.42(17$ months $)$ \\
$25{ }^{\circ} \mathrm{C}$ & 0.190 & 0.9116 & $2.83(34$ months $)$ \\
$2-8^{\circ} \mathrm{C}$ & 0.013 & 0.9197 & $>5$ \\
\hline $\mathrm{E} 40^{\circ} \mathrm{C}, 75 \% \mathrm{RH}$ & 1.530 & 0.1944 & $0.42(5$ months $)$ \\
$25{ }^{\circ} \mathrm{C}$ & 0.275 & 0.9807 & $2.17(26$ months $)$ \\
$2-8^{\circ} \mathrm{C}$ & 0.063 & 0.5811 & $>5$ \\
\hline $\mathrm{F} 40^{\circ} \mathrm{C}, 75 \% \mathrm{RH}$ & 2.169 & 0.9661 & $0.17(2$ months $)$ \\
$25{ }^{\circ} \mathrm{C}$ & 0.404 & 0.9574 & $1.25(15$ months \\
$2-8^{\circ} \mathrm{C}$ & 0.070 & 0.6477 & $4.83(58$ months $)$ \\
\hline
\end{tabular}




\section{CONCLUSIONS}

Only half of the formulations studied were found to be stable for two years at ambient temperature which is their expected expiry date and only one for three years in the same condition. In refrigerator, five formulations were found to be stable for four years or more. One system was unstable in the three conservation conditions studied, including appearance and $\mathrm{pH}$. These results indicate the need for exhaustive stability studies carried out in the pharmaceutical industry.

\section{REFERENCES}

Ahmed HM, Ebeid WB.The use of laser-induced fluorescence or ultraviolet detectors for sensitive and selective analysis of tobramycin or erythropoietin in complex samples. Spectrochim Acta A Mol Biomol Spectrosc, 2015; 143: 12-19.

Brandl M, Gu L.Degradation of tobramycin in aqueous solution. Drug Dev Ind Pharm, 1992; 18(13):1423-1436.

Caturla MC, Cusido E, Westerlund, D. High-performance liquid chromatography method for the determination of aminoglycosides based on automated pre-column derivatization with o-phthalaldehyde. J Chrom A, 1992; 593(1-2):69-72.

Chopra S, Vanderheyden G, Hoogmartens J, Van Schepdael A, Adams E. Comparative study on the analytical performance of different detectors for the liquid chromatographic analysis of tobramycin. J Pharm Biomed Anal, 2010; 53:151-157.

Dash AK, Suryanarayanan R. A liquid-chromatographic method for the determination of tobramycin, J Pharm Biomed Anal, 1991 ; 9(3):237-245

Dash AK, Suryanarayanan R. Solid-State properties of Tobramycin. Pharm Res, 1991b; 8 (9): 1159-1165.

El-Attug MN, Hoogmartens J, Adams E, Van Schepdaela A. Optimization of capillary electrophoresis method with contactless conductivity detection for the analysis of tobramycin and its related substances. J Pharm Biomed Anal, 2012; 58:49-57.

Feng CH, Lin SJ, Wu HL, Chen SH. Trace analysis of tobramycin in human plasma by derivatization and high-performance liquid chromatography with ultraviolet detection. J Chrom B, 2002; 780(2):349-354.

Guo MX, Wrisley L, Maygoo E. Measurement of tobramycin by reversed-phase high-performance liquid chromatography with mass spectrometry detection. Anal Chim Acta, 2006; 571(1):12-16.

Hanko VP, Rohrer JS. Determination of tobramycin and impurities using high-performance anion exchange chromatography with integrated pulsed amperometric detection. J Pharm Biomed Anal, 2006; 40 (4):1006-1012.

Hanko VP, Rohrer JS, Liu HH, Zheng C, Zhang S, Liu X, Tang $\mathrm{X}$. Identification of tobramycin impurities for quality control process monitoring using high-performance anion-exchange chromatography with integrated pulsed amperometric detection. J Pharm Biomed Anal, 2008; 47:828-833.

Hassan S, Zaheer E, Muhammad IN, Hassan A, Ali M, Qadri M. Determination of chemical stability of various Famotidine dosage forms by UV-Visible spectrophotometric method and data analysis by R-GUI stability Software. J Basic Appl Sci, 2015; 11:232-239.
He D, Yang L. Determination of tobramycin sulfate drops by HPLC with post-column derivatization. Chin J Antibiot, 2010; 35 (10): 771-773.

He S, Chen Q, Sun Y, Zhu Y, Luo L, Li J, Cao Y. Determination of tobramycin in soil by HPLC with ultrasonic-assisted extraction and solid-phase extraction. J Chrom B, 2011; 879: 901-907.

International Conference on Harmonization. ICH Q1E Guideline on Evaluation for stability data (2003).

Keevil BG, Lockhart SJ, Cooper DP. Determination of tobramycin in serum using liquid chromatography-tandem mass spectrometry and comparison with a fluorescence polarisation assay. $\mathrm{J}$ Chrom B, 2003; 794:329-335.

Lee H, Wu P. Lee Y. stab: An R package for drug stability data analysis. Comput Methods Programs Biomed, 2010; 100:140-148.

Manyanga V, Elkady E, Hoogmartens J, Adams E. Improved reversed phase liquid chromatographic method with pulsed electrochemical detection for tobramycin in bulk and pharmaceutical formulation. J Pharm Anal, 2013; 3(3):161-167.

Mashat M, Chrystynb H, Clarka BJ, Assia KH. Development and validation of HPLC method for the determination of tobramycin in urine samples post-inhalation using pre-column derivatisation with fluorescein isothiocyanate. J Chrom B, 2008; 869:59-66.

Megoulas NC, Koupparis MA. Development and validation of a novel HPLC/ELSD method for the direct determination of tobramycin in pharmaceuticals, plasma, and urine. Anal Bioanal Chem, 2005; 382 (2): 290-296.

Pfeifer C, Fassauer G, Gerecke H, Jira T, Remane Y, Frontini R, Byrne J, Reinhardt R. Purity determination of amphotericin B, colistin sulfate and tobramycin sulfate in a hydrophilic suspension by HPLC. J Chrom B, 2015; 990:7-14.

Russ H, McClery D, Katimy R, Montana JL, Miller RB, Krishnamoorthy R, Davis CW. Development and validation of a stabilityindicating HPLC method for the determination of Tobramycin and its related substances in an ophthalmic suspension. J Liq Chrom Rel Technol, 1998; 21(14):2164-2181.

Sun N, Mo W M, Shen ZL, Hu BX. Adsorptive stripping voltammetric technique for the rapid determinationof tobramycin on the hanging mercury electrode. J Pharm Biomed Anal, 2005; 38:256-262.

Szúnyog J, Adams E, Roets E, Hoogmartens J. Analysis of tobramycin by liquid chromatography with pulsed electrochemical detection. J Pharm Biomed Anal, 2000; 23(5): 891-896.

The United States Pharmacopeia $38^{\text {th }}$ Ed. (Spanish version) U.S. Pharmacopeial Convention, Rockville, MD; 2015, 6020-6021.

Valentini F, Buldini, PL, Landi E, Tampierini A, Tonelli D. HPLC determination of tobramycin in a simulated body fluid.Microchem J, 2008; 90:113-117.

Zhang Z, Peng J. A simple HPLC method for determination tobramycin in plasma and its application in the study of pharmacokinetics in rats. LatAm J Pharm, 2012; 30(3):563-567.

\section{How to cite this article:}

Rosasco MA, Segall AI. Determination of the Chemical Stability of Various Formulations of Tobramycin Eye-Drops by HPLC Method and Data Analysis by R-GUI Stability Software. J App Pharm Sci, 2015; 5 (12): 008-013. 